\title{
Control System Design for Rotorcraft-based Unmanned Aerial Vehicles using Time-domain System Identification
}

\author{
David Hyunchul Shim ${ }^{*}$, Hyoun Jin Kim ${ }^{*}$, Shankar Sastry ${ }^{* *}$ \\ "Graduate Students, Department of Mechanical Engineering, University of California at Berkeley \\ "*Professor, Department of Electrical Engineering and Computer Science, University of California at Berkeley \\ \{hcshim,jin,sastry\}@ robotics.eecs.berkeley.edu
}

\begin{abstract}
This paper introduces the development of flight control system for rotorcraft-based unmanned aerial vehicle(RUAV). In this research, the linear time-invariant model valid for hover is sought. The system response data is acquired in carefully devised experiment procedure and then a linear time-invariant system model is obtained using time-domain analysis method. The acquired model is used to design feedback controller consisting of inner-loop attitude feedback control, mid-loop velocity feedback control and the outer-loop position control. The proposed controller is implemented on digital onboard computer and tested in a Berkeley RUAV and shows outstanding hovering performance.
\end{abstract}

\section{Introduction}

BErkeley AeRobot (BEAR) project aims to organize multiple number of autonomous agents into integrated and intelligent systems with reduced cognition and control complexity, faulttolerance, adaptivity to changes in task and environment, modularity and scalability to perform complex missions efficiently. In this hierarchical structure, UAVs play an important role as the mobile aerial units to perform given tasks ordered by ground-based command station via wireless communication network. Rotorcraft airframe suits our application particularly well due to their versatile maneuverability such as vertical takeoff/landing, hovering, sideslip, pirouette, and so on.

During the last decade, significant progresses are made on the development for small-size RUAVs[2,3,4,5]. One of the strongest thrusts of this progress was the advent of powerful but small microcomputers and navigation sensors. The computing power of ordinary PC CPU has become sufficient to process vehicle-level real-time discrete-time control as well as high-level trajectory planning and decision making. Strap-down inertial navigation system (INS) units consisting of micromachined inertial sensors are now available at surprisingly smaller size and lower cost. Moreover, the accuracy of INS is remarkably improved using global positioning system (GPS) as the correctional positioning sensor. Advances of the supporting electronics such as compact wireless communication devices and high capacity batteries also contribute to the construction of powerful RUAV less than $10 \mathrm{~kg}$.

The acquisition of high fidelity system model of target UAV is a crucial step towards the successful design of high-performance flight control system. In general, however, it is a challenging process to identify RUAV dynamics due to its multi-input multioutput (MIMO) characteristics, nonlinearity, severe noise and disturbance, and wide flight envelop. Moreover, in RUAV research, there are more limitations on accessing test facilities and resources. Hence, it has been often attempted to borrow full size helicopter models[1] as the template for small-size RUAV dynamics, assuming the dynamics of full-size and RUAV are similar. Unfortunately, this is rather hard to be justified due to the servorotor mechanism found in almost all of small size helicopters. The servorotor, or Bell-Hiller Stabilizer system, has a strong damping effect on the rotor dynamics and significantly delays the overall rotor response. In some other cases, it is attempted to design the flight controllers of simple structure by trial and error during the actual flight. This alternative approach would require only the basic understanding of the plant, but the resulting controller by this process is not likely to be the optimal tuning and it is also a very dangerous practice risking damages to test pilot crews and the costly UAV system.

Therefore, in this paper, we adopt a systematic way to find a dynamics model using time-domain system identification method. Once the models are determined, a multi-loop SISO control structure is designed and implemented on Berkeley RUAVs. The experimental setups and the actual flight test results using the proposed controllers are shown and the results are discussed.

\section{System Identification}

It is well known that a helicopter is a very difficult system for identification due to its unstable, highly nonlinear, time-varying, and MIMO characteristics. The model also varies significantly depending on the flight mode, hovering or forward flight for example, and the measurement is prone to be contaminated by mechanical vibrations. Therefore, we should first decide which mode to identify. In this research, linear time-invariant model is chosen to capture the helicopter dynamics in hover. The order of the model should be chosen depending on the bandwidth of interest. In this research, a model valid for near-hover condition is to be obtained for the design of hovering and low-velocity maneuver.

A 6-degrees-of-freedom linear rigid body helicopter model augmented with first-order approximation of servorotor dynamics is given by a differential equation

where

$$
\dot{\mathbf{x}}=\mathbf{A x}+\mathbf{B u}
$$

$$
\begin{aligned}
& \mathbf{x}=\left[\begin{array}{lllllllllll}
u & \vee & p & q & \Phi & \Theta & a_{l s} & b_{l s} & w & r & r_{f b}
\end{array}\right]^{T} \\
& \mathbf{u}=\left[\begin{array}{llll}
u_{a_{1 s}} & u_{b_{s}} & u_{\theta_{M}} & u_{r_{r e s}}
\end{array}\right]^{T}
\end{aligned}
$$




$$
\begin{array}{cl}
u, v, w: & \text { body-coordinate velocity } \\
\Phi, \Theta, \Psi: \text { roll, pitch, yaw angle, respectively } \\
p, q, r: \quad \text { roll, pitch, yaw rate, respectively } \\
a_{1 s}, b_{1 s}: & \text { flapping angle } \\
r_{f b}: & \text { feedback gyro system state }
\end{array}
$$

One candidate model of eleventh order takes the form of Eq. (4) as suggested by Mettler et al [2]. One distinction of this model is the explicit account for the servorotor, which modifies the helicopter dynamics significantly. Commonly used servorotor for small-size RUAV is Bell-Hiller stabilizer system, which has a pair of paddleshaped blades that are connected to the main blades by a series of mechanical linkages. The most important role of servorotor is to slow down the roll and pitch response so that human pilot on the ground can control the helicopter with remote controller. The dynamics can be described as coupled first order model as following[2]:

$$
\begin{aligned}
& \dot{b}_{1 s}=-\frac{b_{1 s}}{\tau_{f}}-p+B_{a_{1 s}} a_{1 s}+B_{u_{a \mid s}} u_{a_{1 s}}+B_{u_{b \mid s}} u_{b_{1 s}} \\
& \dot{a}_{1 s}=-\frac{a_{1 s}}{\tau_{f}}-q+A_{b_{1 s}} b_{1 s}+A_{u_{a \mid s}} u_{a_{1 s}}+A_{u_{b \mid s}} u_{b_{1 s}}
\end{aligned}
$$

$\tau_{f}$ can be interpreted as the time constant of the servorotor response to the swash plate tilt angles. The body angular rate $p$ and $q$ appear as feedback terms because of the gyroscopic effect of the stabilizer bar. The equations also include the input coupling terms, which are not small as shown in Eq. (4). The angular rate dynamics is coupled with blade flapping angles $\left(a_{1 s}, b_{1 s}\right)$ and body velocities $(u, v)$. Translational body velocity $u$ and $v$ mainly are affected by the sum of the tilt angle and the flapping angles in each roll and pitch direction.

The heave dynamics is approximated by first order quasi-static model. This model yields linear, low-order approximation of the highly nonlinear high order heave dynamics. For higher bandwidth controllers, the third or fourth order of model containing inflow and flapping dynamics should be used [8]. Yaw dynamics is also stable and modeled as first order system with reasonable fidelity. One special feature of yaw dynamics is the built-in feedback action of yaw rate in the loop, which is provided by the commercially available rate gyro amplifier. Even though the uncompensated yaw dynamics is stable, the variation of the antitorque of the main rotor continually perturbs the heading of the helicopter. The yaw rate feedback counteracts the torque by compensating the tail rotor collective pitch and is chosen to remain in the system for manual flights. The gyro system model is also suggested by Mettler [2] and it is very effective to capture the dynamics with high accuracy, as shown in Figure 1 . One deficiency of the model (4) is the absence of the cross-coupling from yaw to sideslip and roll. It can be additionally parametrized in the model, but it turned out during the numerical process that the additional parameters are cumbersome to find because they appear as a product of parameters and the numerical process becomes singular.

$$
\mathbf{A}=\left[\begin{array}{ccccccccccc}
-0.0629 & 0 & 0 & 0 & 0 & -g & -g & 0 & 0 & 0 & 0 \\
0 & 0.0305 & 0 & 0 & g & 0 & 0 & g & 0 & 0 & 0 \\
0.2978 & -0.7061 & 0 & 0 & 0 & 0 & 40.361 & 237.42 & 0 & 0 & 0 \\
1.3057 & -1.2199 & 0 & 0 & 0 & 0 & 220.18 & -11.438 & 0 & 0 & 0 \\
0 & 0 & 1 & 0 & 0 & 0 & 0 & 0 & 0 & 0 & 0 \\
0 & 0 & 0 & 1 & 0 & 0 & 0 & 0 & 0 & 0 & 0 \\
0 & 0 & 0 & -1 & 0 & 0 & -4.3459 & 1.4487 & 0 & 0 & 0 \\
0 & 0 & -1 & 0 & 0 & 0 & -1.5915 & -4.3459 & 0 & 0 & 0 \\
0 & 0 & 0 & 0 & 0 & 0 & -3.0523 & -15.063 & -1.3453 & 0.2222 & 0 \\
0 & 0 & -0.0178 & 0 & 0 & 0 & 0 & 0 & 1.1860 & -2.9986 & -22.126 \\
0 & 0 & 0 & 0 & 0 & 0 & 0 & 0 & 0 & 3.1541 & -9.5035
\end{array}\right]
$$

Table 1. Eigenvalues of the identified helicopter system

\begin{tabular}{|c|c|}
\hline Mode & Value \\
\hline Phugoid 1 & $-0.3184 \pm 0.5157 \mathrm{j}$ \\
\hline Phugoid 2 & $0.0087 \pm 0.5675 \mathrm{j}$ \\
\hline Roll & $-2.4425 \pm 15.6953 \mathrm{j}$ \\
\hline Pitch & $-2.1723 \pm 14.9316 \mathrm{j}$ \\
\hline Yaw & $-6.2449 \pm 7.6990 \mathrm{j}$ \\
\hline Heave & -1.3134 \\
\hline
\end{tabular}


Since the model is treated as a linear model, the nonlinearity of helicopter aerodynamics should not be excited by the excessive amount of control action. Hence, the appropriate design of control input signals for flight test is extremely important for the identification of target flight dynamics. A number of experimental flights have been made to collect the flight data and the pilot input at $50 \mathrm{~Hz}$ sampling rate. The control input consists of the combination of frequency-sweeping and random signals in longitudinal, lateral, yaw and heave channels in turn. At the first stage, the control in longitudinal and lateral channels are given simultaneously to capture the coupling between these axes while other channels are controlled to maintain constant altitude and heading. In the next stage, main rotor collective pitch or tail collective pitch is mainly perturbed. Finally, control signals are issued in all channels to capture the cross-coupling term.

Before processing the data, the angular rate signals are filtered by zero-phase noncausal discrete-time filters to filter out high frequency noise without introducing phase delay. The experiment result is processed using time-domain output-error minimization tool from MatLAB ${ }^{\mathrm{TM}}$ System Identification Toolbox ${ }^{\mathrm{TM}}$. The prediction error method (PEM) is an estimation algorithm, which seeks to a set of parameters minimizing the quadratic error between the predicted output and experiment data [6,9]. It should be noted that this method is extremely sensitive to the initial guess of the parameters and easily trapped in local minima of the parameter hypersurface. To obtain meaningful results other than some parameter set that blindly matches the time history, the following technique is devised. First, the angular dynamics augmented with rotor dynamics is identified using initial guess. Since the angular rate/rotor dynamics is known to be stable and small number of parameters are involved, the numerical solution converges to consistent solutions. Then the horizontal dynamics, i.e., the longitudinal and lateral dynamic with linear velocity terms $u$ and $v$ are identified while fixing the angular dynamics parameters. This stage is rather challenging due to the unstable linear velocity dynamics. Shorter length of experiment data should be used to avoid the instability of the predictor and the divergence of the prediction error with the small mismatch of the initial condition and parameters. The solution is found after a large number of iterations using the experimental data from different time intervals. Separate from the longitudinal and lateral dynamics, the heave and yaw dynamics is identified in similar manner. The inherent stability of yaw and heave allows nice convergence of the parameters. Once these two subsystems are identified, they are combined as the full-model dynamics and then the cross-coupling terms are estimated. Finally, a small number of iteration is performed to recalibrate the parameters in the subsystems.

\section{Controller Design and experiment}

Based on the identified model found above, stabilizing control law is designed. As our first approach, we employ multi-loop singleinput, single-output (SISO) control structure. Classic control design approach has a number of advantages such as simple structure, straightforward design process and low computing load imposed on the flight control system. However, the overlooked cross-coupling can cause undesirable parasite response. Currently decoupling control algorithm is being developed.
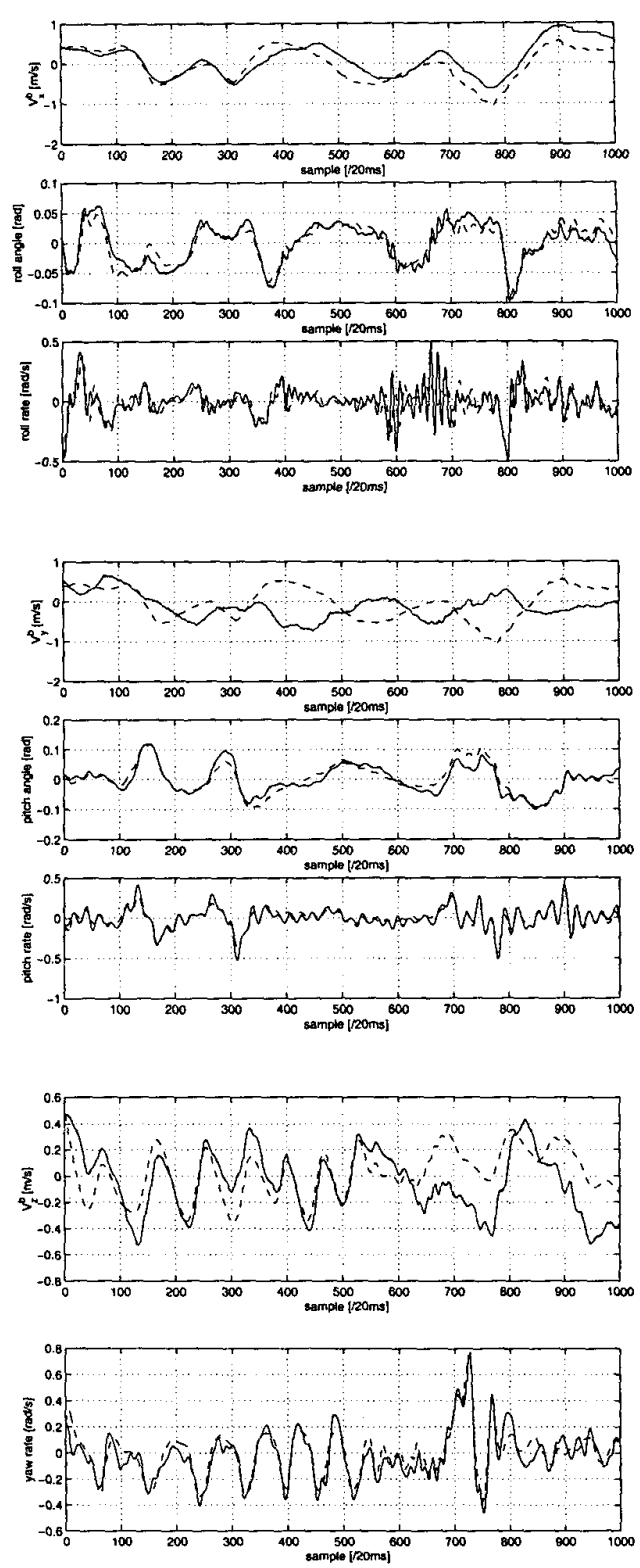

Figure 1. The original response and estimated response of the identified model (solid: original; dashed: estimates)

The longitudinal and lateral dynamics are further decoupled into two SISO systems ignoring the rather large cross-coupling effect and then the compensators for each loop is designed. The attitude dynamics is modeled as the cascade form of single integrator at each output of the angular rate dynamics. Since the servorotorangular rate dynamic subsystem is inherently stable, the attitude dynamics can be stabilized by simple proportional feedback compensation. This is a great advantage because the noisy angular rate signal is not necessary to be fed back for stabilization. The 
adequate gains for all loops are determined using standard root locus method for acceptable response speed and damping ratio.

After the innermost loop gain is determined, the gain for linear velocity feedback loop is found. The root locus analysis for this middle loop shows that proportional feedback is sufficient to stabilize the linear velocity loop. Finally, the position loop gains for $\mathrm{x}$ and $\mathrm{y}$ channel are determined in the similar manner.

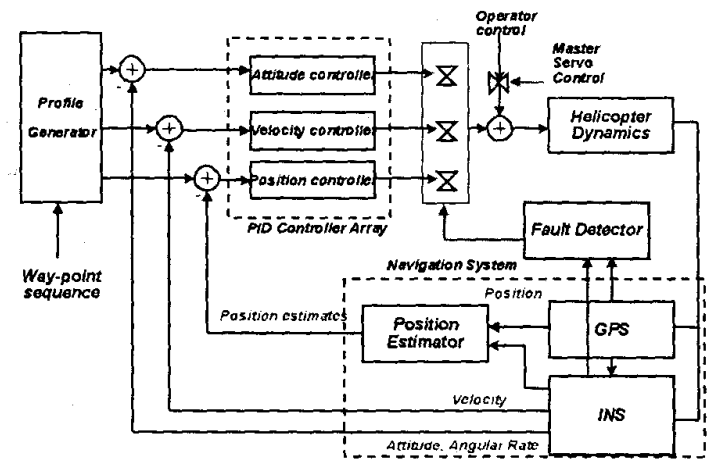

Figure 2. The proposed controller architecture using SISO multiloop controllers

This classical approach enables simple, but very effective control algorithm, as shown in Figure 2. The control structure can realize a couple of flight modes by switching some parts or all of the loops on and off. In cruise mode, the velocity and attitude loops are closed for stabilization and tracking. When hovering over a certain spot is required, the outmost loop for position feedback is closed along with the inner loops. This algorithm is programmed in flight controller and showed the effectiveness in a series of autonomous flight. This structure is also very useful to integrate the fault detection functionality in the loop. One common problem in the sensor system is the fragile GPS lock-up of NovAtel RT-2. When RT-2 loses the $2 \mathrm{~cm}$ accuracy, the position estimate degrades to $20 \mathrm{~cm}$ or worse, causing the position and velocity solutions of INS to jump. When the fault-detector detects this event, it disengages the position controller first and then velocity controller next as the position and velocity estimates degrade further. Since the attitude estimation is affected by faulty GPS operation later than position and velocity estimation, the attitude loop is retained while waiting for GPS to return to normal operation or manual recovery is attempted otherwise.

\section{Experiment}

Berkeley UAV research test bed is established in the perspective of multiple-UAV/UGV operations. Base airframes are chosen from those limited small-size hobby or industrial purpose helicopters in the market. For base airframe, BEAR has chosen four different sizes of model helicopters: Yamaha industrial helicopter RMAX and R-50, Bergen Industrial Twin, and Kyosho Concept 60. Kyosho Concept 60 Graphite is the base airframe used in this research because of its low-cost, small-size, and easily maintainable body. The actual RUAV is shown in Figure. 3.
An RUAV consists of the base airframe and the integrated avionics systems, which can be categorized into the following: 1) flight control computer (FCC), 2) navigational sensors, 3) communication module, and 4) onboard power pack.

Flight control computer (FCC) is constructed using PC104compatible boards due to their industry-proven reliability, compact size and expandability. Due to the space and weight constraint of Ursa Minor 3, an RUAV based on Kyosho Concept 60 , the main board has Cyrix MediaGX 233MHz CPU board with 64MB RAM and 40MB FlashRAM for booting. Serial port expansion board, custom take-over board (TOB), and DC-DC conversion power supply board are added to complete a fully functioning flight control system. As the heart of the navigation sensor, Boeing DQI-NP inertial navigation system (INS) is adopted. DQI-NP consists of a pack of solid state inertial sensors and embedded digital signal processors with serial port. DQI-NP needs periodic position update from additional GPS to correct the diverging position error. The global positioning system (GPS) system used in this research is NovAtel RT-2, which has incredible accuracy of $2 \mathrm{~cm}$. The flight control computer acquires the position, linear/angular velocity and attitude from DQI-NP, and high accuracy position estimate from NovAtel RT-2 via RS232. It also updates the DQI-NP with the position measured by GPS at every second. Based on the acquired navigation data, FCC computes the control output for four channels: main rotor collective pitch, tail rotor collective pitch, main rotor longitudinal cyclic pitch, and lateral cyclic pitch. These control surfaces are actuated by commercially available small-size servos, which accept PWM signal (14-21 ms period, $0.8-2.4 \mathrm{~ms}$ on duty) as command. The output angle of servo rotor is proportional to the on-duty duration. The PWM signal is generated by Intel 8254 counter/timer chip. To ensure safety, a special circuit is added on the TOB to switch from FCC control to human pilot command by a toggle switch on the radio transmitter. Other channels of counter/timer board read the radio receiver output to log the human pilot's command, which is extremely valuable for system identification and feedback-assisted flight. The helicopter engine is also regulated at the constant RPM (13000 RPM) using commercial hobby-purpose engine governor.

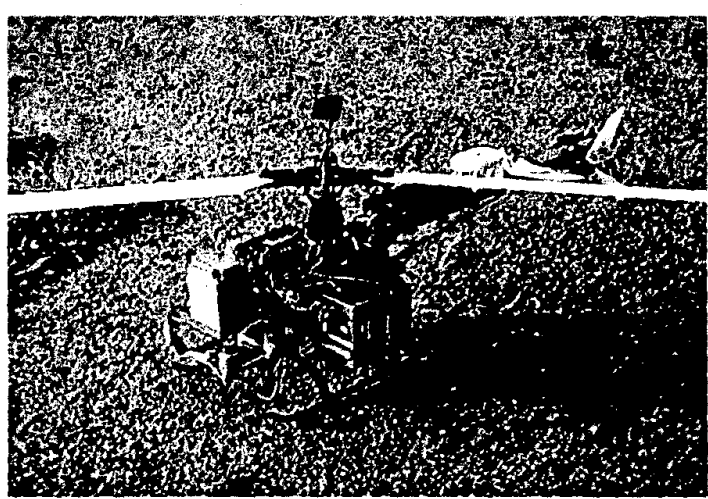

Figure 3. One of Berkeley UAVs: Ursa Minor 3

The communication module contains two $900 \mathrm{MHz}$ wireless modem cards. One $900 \mathrm{MHz}$ wireless modem serves as the communication link to receive the differential GPS (DGPS) 
correction information from the ground station GPS system. The FCC communicates with the ground station via wireless modem. The wireless modem is very convenient to program, but has limited bandwidth (up to $115.2 \mathrm{kbps}$ ). In larger RUAVs, wireless Ethernet is the standard data communication channel, which supports higher bandwidth (>1Mbps) and peer-to-peer networking environment. Ground monitoring station consists of a portable computer connected with a communication device such as wireless modem or wireless Ethernet. The ground station monitors and stores the flight data of the UAV and also sends the navigation commands such as sequences of waypoints.

Table 2. Specifications of Ursa Minor 3

\begin{tabular}{|c|c|c|}
\hline \multirow{2}{*}{ Dimension } & Height & $0.47 \mathrm{~m}$ \\
\cline { 2 - 3 } & Width & $0.39 \mathrm{~m}$ \\
\cline { 2 - 3 } & Length & $1.4 \mathrm{~m}$ \\
\hline Rotor & Diameter 1.5m \\
\hline Weight & $1300 \mathrm{RPM}$ \\
\hline \multirow{2}{*}{ Engine } & $4.5 \mathrm{~kg}$ airframe \\
& $4.8 \mathrm{~kg}$ avionics \\
\hline & OS FX91 \\
& 2cycle glow engine \\
& $2.8 \mathrm{hp}$ \\
\hline \multirow{3}{*}{ Onboard System } & $\begin{array}{c}\text { PC104 based flight computer } \\
\text { Boeing DQI-NP IMU } \\
\end{array}$ & NovAtel GPS \\
& Two wireless modems \\
\hline
\end{tabular}

Experiment is performed as follows. The RUAV is first placed at the test field and then the onboard system is turned on. The GPS system automatically starts tracking the GPS satellite signals to compute the current location. The DGPS broadcast are also used to achieve the ultimate accuracy of $2 \mathrm{~cm}$. Once GPS is completely locked on, the DQI-NP system is initialized and sequenced into the fine-alignment mode. During this process, the DQI system should be left undisturbed for the correct estimation of the scale and bias factors of the six solid state inertial sensors. After these two navigation sensors are initialized, the engine is started manually and the rotor is controlled to about $90 \%$ of the hovering RPM to avoid any low frequency mechanical resonance degrading the accuracy of INS and GPS measurements. Then the test pilot engages the attitude/velocity controller using a switch on the transmitter and then takes the helicopter off from the ground. At this time, only the steady heave reference command is given. During the repeated experiments, the attitude-velocity controller showed its stable performance over the entire operating range of the engine and it promises reliable and accurate automatic take-off and landing control. Once the helicopter reaches the desired altitude, the hovering controller, i.e., position/velocity/attitude loop controller, is activated by pilot. Figure 4 shows one of the experiment results performed by Ursa Minor 3. In this session, the RUAV showed more than 3 minutes of continuous hovering using the hovering controller. As found in the graph, the RUAV hovers within $30 \mathrm{~cm} \times 30 \mathrm{~cm} \times 30 \mathrm{~cm}$ cube for more than 60 seconds while maintaining the constant heading within \pm 1 degree. Oscillatory motions in all $x, y, z$ and heading are attributed to the uncompensated coupling among the longitudinal, lateral, yaw, heave modes.
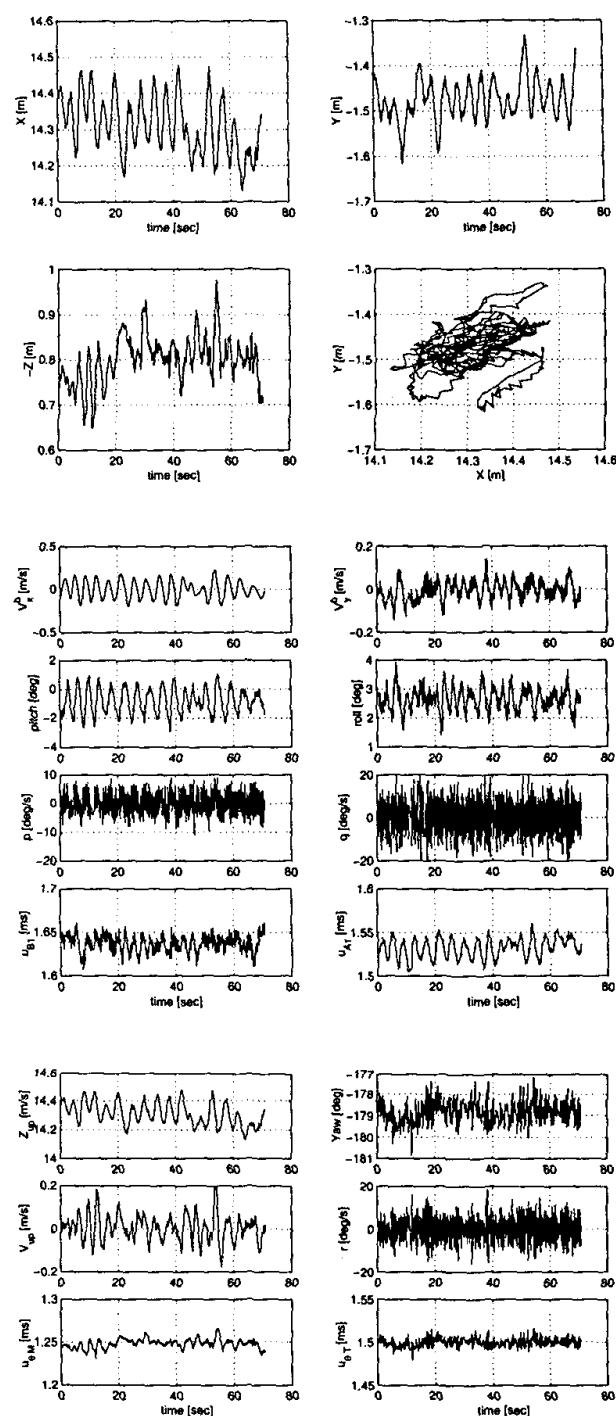

Figure 4. The experiment data of Ursa Minor 3 in hover by multiloop SISO control

\section{Conclusion}

This paper introduced the development of high performance RUAV controller design at University of California at Berkeley. The rotor dynamic model proposed by Mettler matches the servorotor dynamics particularly well for Berkeley RUAVs also. The obtained linear time invariant (LTI) model is used to design SISO proportional feedback compensators in tri-loop configuration. The proposed controller structure exhibits outstanding hovering performance with tolerable level of oscillatory motion. However, the high level of coupling between longitudinal and lateral dynamics calls for more elegant MIMO approach such as $\mathrm{H}$-infinity control or $\mu$-synthesis control methods. Currently, MIMO design methodology is also applied to 
the helicopter control and this result will be published in the near future.

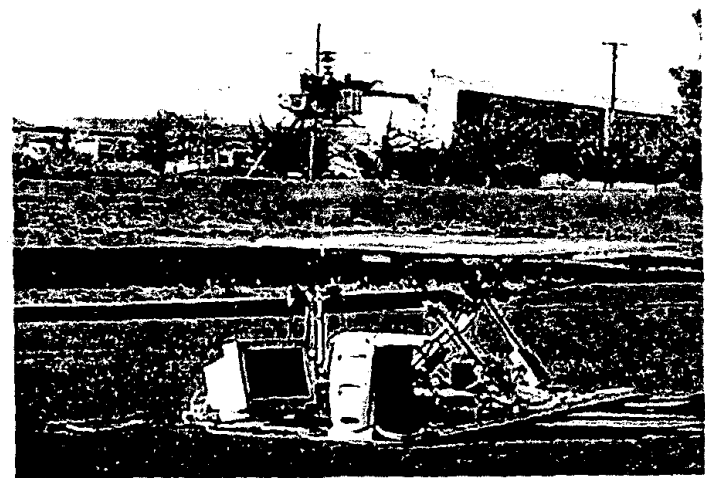

Figure 5. Ursa Minor 3 hovering over the ship deck motion platform under automatic hovering control

\section{Acknowledgement}

This research was supported in part by the Army Research Office DAAH04-96-1-034l and by the Office of Naval Research under Grants N00014-97-1-094.

\section{Reference}

[1] R.W. Prouty, Helicopter Performance, Stability and Control, Krieger Publishing Company, 1995.

[2] B. Mettler, M. B. Tischler, T. Kanade, "System Identification of Small-Size Unmanned Helicopter Dynamics," American Helicopter Society $55^{\text {th }}$ Forum, Montreal, Quebec, Canada, May 1999.

[3] H. Shim, T. J. Koo, F. Hoffmann, S. Sastry, "A Comprehensive Study of Control Design for an Autonomous Helicopter," $37^{\text {h }}$ IEEE Conference on Decision and Control, pp. 3653-3658, 1998

[4] C. P. Sanders, P. A. DeBitetto, E. Feron, H. F. Vuong, N. Leveson, "Hierarchical Control of Small Autonomous Helicopters," $37^{\text {th }}$ IEEE Conference on Decision and Control, pp. 3629-3634, 1998

[5] J. E. Corban, A. J. Calise, J. V. R. Prasad, "Implementation of Adaptive Nonlinear Control for Flight Test on an Unmanned Helicopter," $37^{\text {th }}$ IEEE Conference on Decision and Control, pp. Pp. 3641-3646, 1998.

[6] L. J. Ljung, Matlab System Identification Toolbox User's Guide, The Math Works, Inc., 1997.

[7] Anon., Rotorcraft System Identification, AGARD Advisory Report, 1991.
[8] R. T. N. Chen, M. B. Tischler, "The Role of Modeling and Flight Testing in Rotorcraft Parameter Identification," The 1986 AHS Forum, System Identification Session, Washington, D.C., 1986.

[9] T. Soderstrom, P. Stoica, System Identification, Prentice-Hall, 1989. 